\title{
Bildfilter als Analysewerkzeug im Technologiesektor von Industrie 4.0
}

\author{
F. Albers ${ }^{1}$, T. Bartsch ${ }^{2}$, D. Kowalke ${ }^{3}$, S. Sade ${ }^{4}$
}

\section{Zusammenfassung}

Der Einrichtprozess der Wasserstrahlschneidanlage erfolgt überwiegend als manueller Prozess. Die fehlende Automatisierung führt zu einem hohen Zeitverlust und der dauerhaften Anwesenheit einer Fachkraft. Dieser Umstand wird durch eine Steigerung des Automatisierungsgrades angegangen. Die Steigerung wird durch die Integration einer Umfeldsensorik und die Erstellung eines Algorithmus realisiert. Dieser erkennt die Halbzeuge im Arbeitsraum, um diese anschließend in ein CAD-Modell zu transformieren.

\section{Stichwörter}

Automatisierung, Bildverarbeitung, Industrie 4.0, Merkmalsfilter, Wasserstrahlschneiden

\section{Problemstellung \& Ausgangslage}

Aufgrund des hohen manuellen Einrichtaufwands wird ein Algorithmus entwickelt, welcher durch Bildverarbeitung den Einricht- und Bedienungsprozess an einer Wasserstrahlschneidanlage, siehe Bild 1, optimiert. Die Optimierung führt zu einer Zeitersparnis, da der zu erstellende Algorithmus automatisiert die Halbzeuge im Arbeitsraum erkennt und diese als CAD-Datei ausgibt. Zum jetzigen Zeitpunkt müssen diese Dateien von Hand, mit erheblichem Zeitaufwand, erstellt werden.

Eine Umfeldsensorik, in Form einer USB-Kamera, wurde zur Bildgenerierung über dem Maschinenbett implementiert. Die damit erzeugten Bilder stellen die Basis dieses Artikels dar und sind der Ausgangspunkt für alle Manipulationsoperatoren.

In einem früheren Abschnitt des Projektes wurden verschiedene lineare und nichtlineare Merkmalsfilter hinsichtlich ihres Aufbaus und mathematischen Funktionsprinzips untersucht. Der Fokus der Betrachtung war die Reduktion eines Bildes auf seine Kanten, da diese zu den auffälligen Bildmerkmalen gehören. Diese bilden eine geeignete Basis zum Erstellen eines Polygonszuges [1]. Dieser Polygonzug fungiert folgend als Grundlage für das Erzeugen der CAD-Datei. Eine stetige und realgetreue Darstellung der Halbzeugkanten ist dementsprechend unerlässlich für alle Folgeschritte. Der Fokus dieses Artikels liegt in der vollständigen Identifikation der Halbzeugkanten.

\footnotetext{
$1 \quad$ B. Eng. Franziska Albers, TH Ostwestfalen-Lippe, Lemgo

2 Prof. Dr. -Ing. Thomas Bartsch, TH Ostwestfalen-Lippe, Lemgo

$3 \quad$ M. Sc. Dennis Kowalke, TH Ostwestfalen-Lippe, Lemgo

$4 \quad$ M. Sc. Sercan Sade, TH Ostwestfalen-Lippe, Lemgo
} 


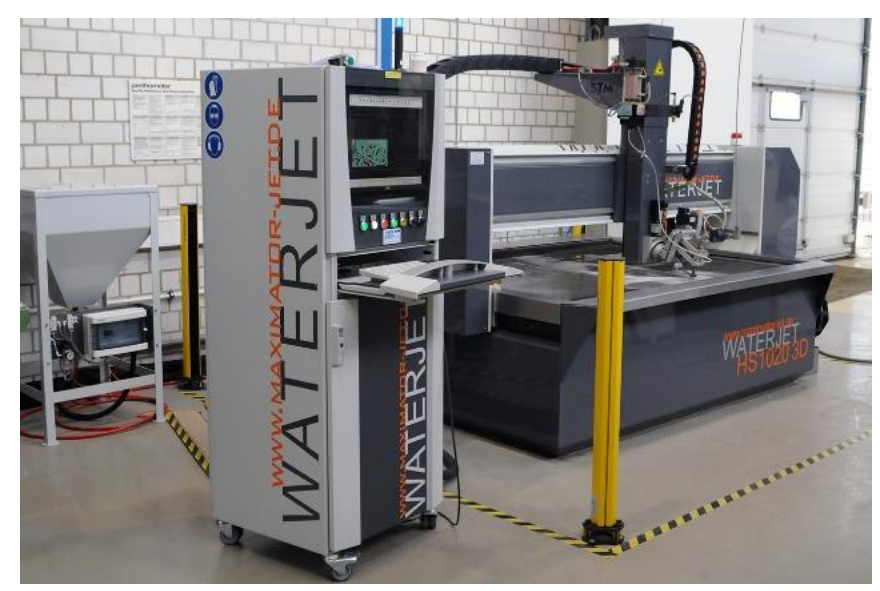

Bild 1: Wassserstrahlschneidanlage

Quelle: [2]

\subsection{Störgrößen}

Der Arbeitsraum der Anlage ist ein mit metallischen Streben durchzogenes Wasserbecken. Die Streben dienen der Ablage der zu bearbeitenden Halbzeuge und haben den Nebeneffekt ein hohes Reflektionsvermögen zu besitzen. Neben den metallischen Streben ist das Wasserbecken selbst eine Störgröße, da dieses ebenfalls eine Reflektionsquelle darstellt. Die Reflektionserscheinungen, sowohl der Streben wie auch der Wasseroberfläche, sind abhängig von dem Wasserstand im Arbeitsraum, wie auch von den umliegenden Lichtquellen. Zudem ist es möglich, dass die Halbzeugoberflächen durch das Wasserbecken benetzt werden. Neben den örtlichen gegebenen negativen Einflussgrößen spielt auch die Werkstoffauswahl eine Rolle. Die Anlage ist in der Lage diverse nichtmetallische und metallische Werkstoffe zu bearbeiten. Dabei sind beispielsweise Werkstoffdicken von:

- $50 \mathrm{~mm}$ bei gehärteten Stählen

- $120 \mathrm{~mm}$ bei Nichteisenmetallen

- $140 \mathrm{~mm}$ bei Verbundstoffen \& Granit/Marmor

schneidbar [3]. Die Vielfältigkeit der Werkstoffe zeigt sich auch in dem Reflektionsverhalten der Halbzeuge. Je nach Werkstoffauswahl ist folglich die Intensitätsverteilung des Bildes unterschiedlich. Insgesamt ergeben sich somit folgende Störgrößen:

- Variable Beleuchtungssituation

- Reflexion Wasseroberfläche

- Reflexion Lamellen

- Wasserstand im Arbeitsraum

- Werkstoffdiversität

- Benetzung der Halbzeuge

\subsection{Filterergebnis vor der Algorithmenoptimierung}

Bei der Betrachtung der verschiedenen Merkmalsfilter wurden verschiedene Filter wie Prewitt, Sobel, Laplace, Canny, Roberts-Cross und Kirsch untersucht. Bei einem Vergleich anhand der Gesichtspunkte:

- Programmumfang

- Programmkomplexität 
- Störanfälligkeit

- Genauigkeit

gewann der Kirschfilter. Nicht nur die eigens durchgeführte Versuchsreihe, sondern auch aktuelle Studien, im Bereich der Medizin und der dynamischen Kantendetektion, verweisen auf die Stärken des Kirschoperators [4-6]. Der Operator selbst besteht aus acht Filtermasken, die in Gleichung 1 dargestellt sind. Zwischen den einzelnen Masken wird jeweils um 45 Grad weitergedreht, so dass insgesamt eine vollständige Umdrehung von 360 Grad erreicht wird. Das Grundprinzip dieses Aufbaus ist, dass je stärker ein Filter auf Kanten reagiert, desto stärker ist auch seine Richtungsabhängigkeit [7]. Die Richtungsabhängigkeit äußert sich im Winkelbereich, welcher hier pro Maske 45 Grad beträgt. Zum Vergleich besteht der Prewitt-Filter lediglich aus zwei Masken, eine für die Horizontale und eine für die Vertikale.

$$
\begin{array}{rlrl}
K_{0} & =\left(\begin{array}{ccc}
5 & 5 & 5 \\
-3 & 0 & -3 \\
-3 & -3 & -3
\end{array}\right) & K_{1}=\left(\begin{array}{ccc}
5 & 5 & -3 \\
5 & 0 & -3 \\
-3 & -3 & -3
\end{array}\right) & K_{2}=\left(\begin{array}{ccc}
5 & -3 & -3 \\
5 & 0 & -3 \\
-3 & -3 & -3
\end{array}\right) \\
K_{3}=\left(\begin{array}{ccc}
-3 & -3 & -3 \\
5 & 0 & -3 \\
5 & 5 & -3
\end{array}\right) & K_{4}=\left(\begin{array}{ccc}
-3 & -3 & -3 \\
-3 & 0 & -3 \\
5 & 5 & 5
\end{array}\right) & K_{5}=\left(\begin{array}{ccc}
-3 & -3 & -3 \\
-3 & 0 & 5 \\
-3 & 5 & 5
\end{array}\right) \\
K_{6}=\left(\begin{array}{ccc}
-3 & 5 & 5 \\
-3 & 0 & 5 \\
-3 & -3 & 5
\end{array}\right) & K_{7}=\left(\begin{array}{ccc}
-3 & 5 & 5 \\
-3 & 0 & 5 \\
-3 & -3 & -3
\end{array}\right) &
\end{array}
$$

Beim Anwenden des Kirschfilters auf eine Aufnahme des Arbeitsraumes bei Tageslicht, entsteht das Filterergebnis aus Bild 2. Das ungefilterte Bild ist in Bild 3 aufgezeigt.

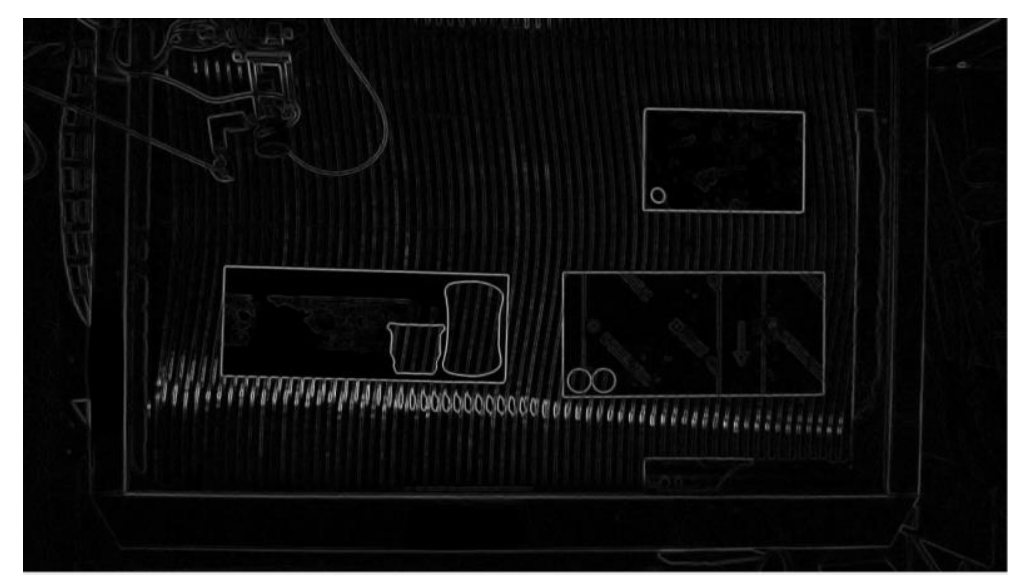

Bild 2: Filterantwort Kirschoperator bei Tageslicht

Quelle: eigene Darstellung erstellt mittels Matlab

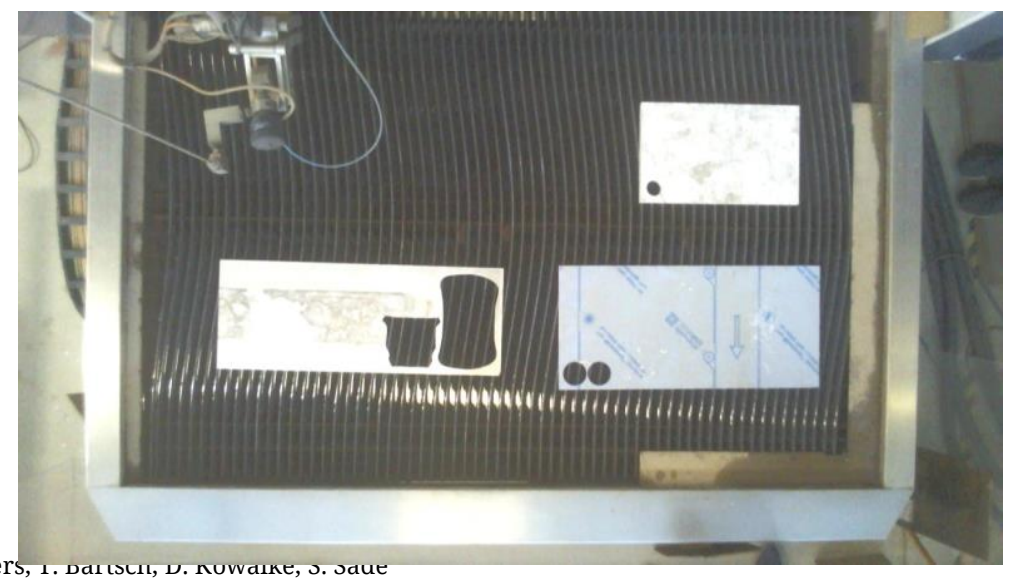


Bild 3: Arbeitsraum bei Tageslicht - ungefiltert Quelle: Umfeldsensorik

Die in Bild 2 dargestellte Filterantwort, zeigt die Kanten von allen Objekten, eingeschlossen Lamellen, Schneidkopf und Wasserreflexionen. Die Funktionalität des Kantenoperators ist somit gegeben und die Herausforderung besteht in der Reduktion des Merkmalsbildes auf die Halbzeuge. Auffällig ist die Verschmelzung der erkannten Halbzeugkanten mit den Störeinflüssen der Lamellen und der Spiegelungen. Ebenso zeigt die Grauwertdifferenz die Intensität einer Kante. Dem Beobachter ist es demzufolge möglich, die Kanten des Bildes zu vergleichen. Ein ähnlicher Grauton im Filterergebnis bedeutet eine ähnliche Kantenstärke. Dies dient nur der ersten Einschätzung und nicht der Weiterverarbeitung, bei dieser wird mit der Bildmatrix gearbeitet und den expliziten numerischen Grauwerten. Ziel ist eine kontrollierte Isolation der Halbzeuge vom restlichem Merkmalsbild. Diese Abtrennung der Halbzeuge ist robust zu gestalten, so dass die Veränderung der Lichtsituation, wie zum Beispiel ein verringertes Sonnenlicht, nicht zu einem Scheitern des Algorithmus führt.

\section{Vorgehensweise}

Die Halbzeugidentifikation ist durch ein zu erstellendes Programm zu erreichen. Die hardwaretechnische Lösung mittels Blenden, Polarisationsfilter oder weiterer Sensorik wird in diesem Artikel nicht thematisiert. Für zukünftige Betrachtungen ist dieser Zweig aber nicht ausgeschlossen. Als Programmierumgebung wird Matlab verwendet.

\subsection{Bildaufbereitung vor der Filterung}

Bild 3 stellt ein RGB-Bild des Arbeitsraumes dar, welches mit einer HD-USB-Webcam aufgenommen wurde. Bild 2 hingegen ist ein Graustufenbild. Der Operator ist nur auf ein Graustufenbild anzuwenden, weshalb die Reduktion des Bildtensors auf eine Bildmatrix, für diesen Anwendungsfall, notwendig ist und so automatisch das Datenvolumen verringert. Neben der Wandelung des Bildes in ein Graustufenbild, ist das Wegschneiden der Peripherie ein weiterer Bearbeitungsschritt, welcher das Datenvolumen verringert ohne, aufgabenbezogen, benötigte Information zu verlieren. Wird das Bild 3 in ein Graustufenbild überführt und zugeschnitten, ändert sich der Bildtensor von $720 \times 1200 \times 3$, was 2.592.000 Matrixelementen entspricht, zu einer Bildmatrix von 670 x 1091. Diese Bildmatrix besteht aus 730.970 Elementen und bedeutet im Vergleich zum Eingangsbild eine Datenreduktion von 71,8 \%. Das Ergebnis dieser Aufbereitung ist Bild 4 zu entnehmen. In allen folgenden Filter- und Manipulationsschritten wird das Eingangsbild zunächst wie aufgezeigt aufbereitet. Bild 4 wird im Folgenden, soweit nicht anders dargelegt, als Eingangsgröße genutzt

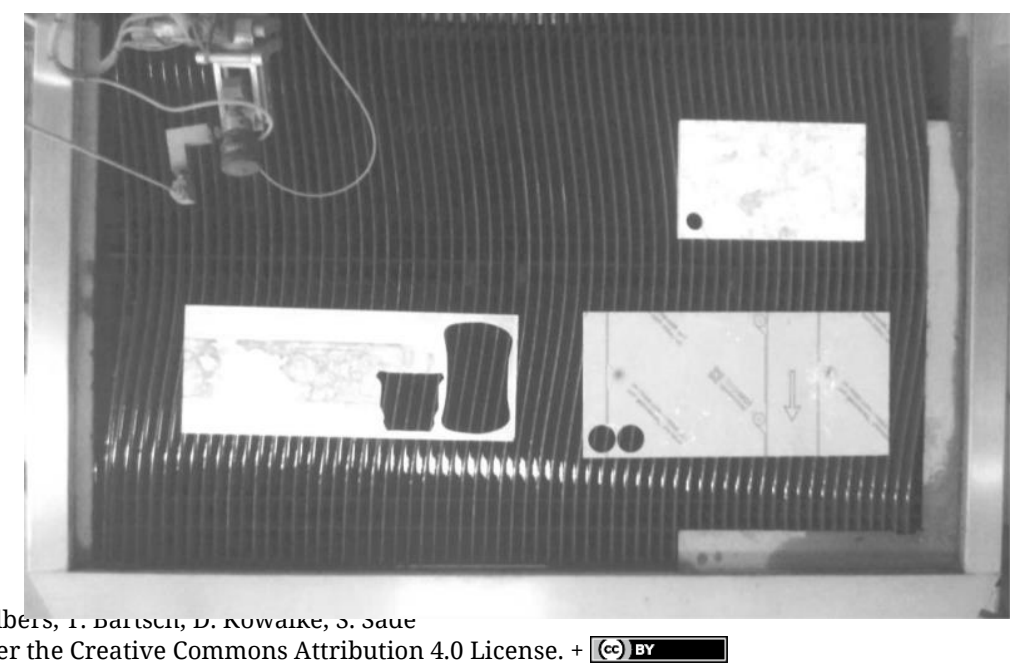

Open Access. (c) 2021, F. Albets,
This work is licensed under the Creative Commons Attribution 4.0 License. + (cc) BY 
Bild 4: Aufbereitetes Eingangsbild

Quelle: eigene Darstellung erstellt mittels Matlab

\subsection{Schwellwertverfahren}

Das Schwellwertverfahren gehört zu den direkten Manipulationsmöglichkeiten im Bild, da aktiv Grauwerte in der Bildmatrix geändert werden. Bei einem klassischen Graustufenbild liegt der Wertebereich von 0 bis 255 zur Verfügung [7]. Bei dem Verfahren wird ein Grauwert entweder herabgesetzt, wenn er unter der Schwelle liegt oder analog beim Überschreiten hochgesetzt. Die Reduktion des Wertes ist dann bis zum Wert null möglich und ist als „Auslöschen“ zu verstehen, da der Grauwert null visuell der Farbe schwarz entspricht [7]. Durch die Verschiebung des Grauwertes nach unten bis auf null, wird analog der Grauwert beim Hochsetzen auf 255 verschoben, was die Farbe Weiß verkörpert. Durch die Anwendung des Verfahrens und der beschriebenen Manipulationen wird künstlich ein Schwarzweißbild erzeugt.

Die Herausforderung an diesem Verfahren ist das Bestimmen des richtigen Schwellwertes. Es existieren bei der Wahl des Schwellwertes drei Möglichkeiten.

Erstens der Wert liegt zu niedrig, siehe Bild 5, wodurch Graubereiche fälschlicherweise als Kante interpretiert werden. Zweitens der Wert ist zu hoch, siehe Bild 6, wodurch relevante Bildbereiche unterdrückt werden und drittens der Schwellwert ist optimal. Optimal bedeutet in diesem Zusammenhang, dass die Halbzeuge vollständig, inklusive ihrer internen individuellen Ausschnitte, angezeigt werden.

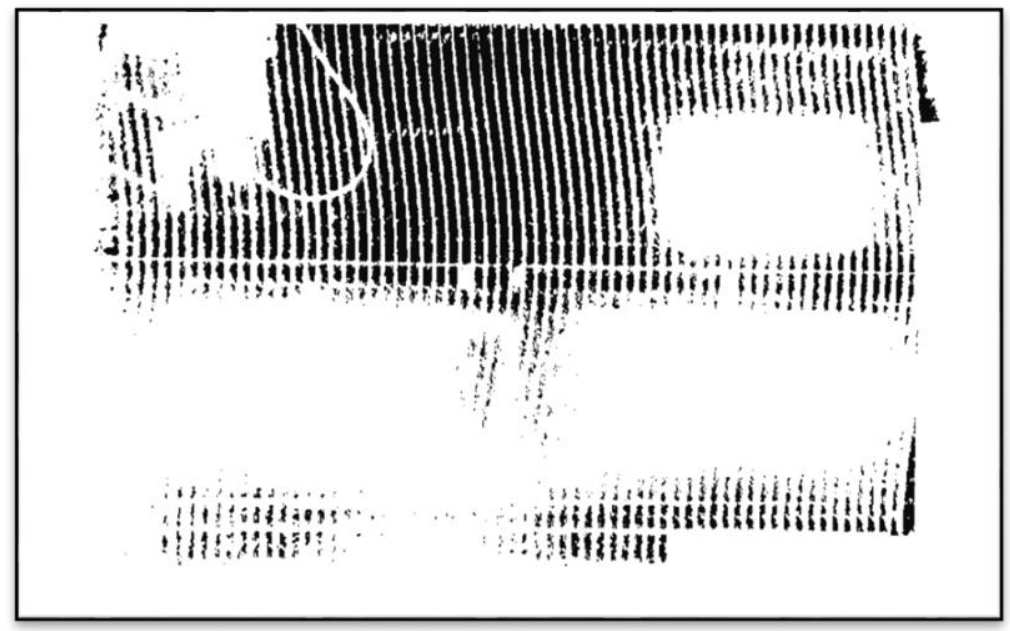

Bild 5: Grauwertmanipulation mit einem Schwellwert von 80 (äußerer Rahmen zur besseren Separation vom Hintergrund hinzugefügt)

Quelle: eigene Darstellung erstellt mittels Matlab

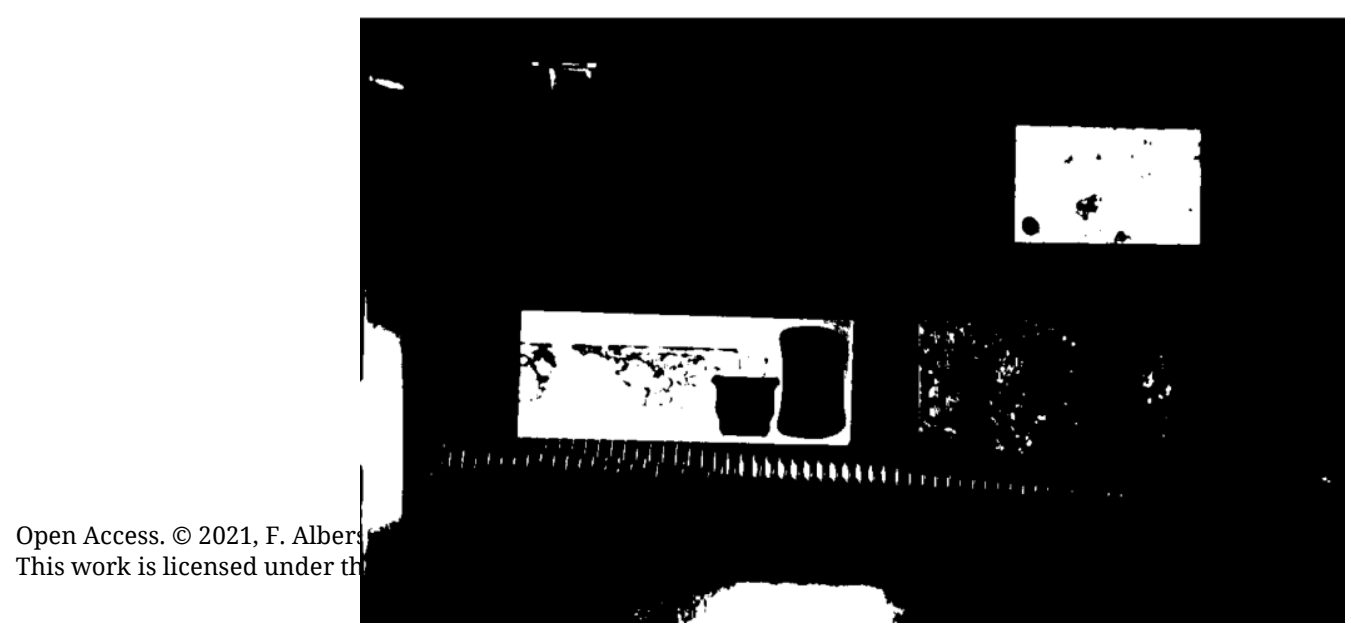


Bild 6: Grauwertmanipulation mit einem Schwellwert von 235

Quelle: eigene Darstellung erstellt mittels Matlab

Anhand von Bild 5 und Bild 6 ist ersichtlich, dass es den optimalen Schwellwert nicht gibt. Selbst bei dem zu hohen Schwellwert von 235 sind Reflexionsartefakte der Wasseroberfläche noch erkannt worden, wobei das rechte untere Halbzeug schon größtenteils unterdrückt wurde. Der perfekte Schwellwert ist daher als Kompromiss zu verstehen, bei welchem das Maximum an unnötigen Bildelementen rausgefiltert wird, ohne dabei fehlerhafte Änderungen an Halbzeugen zu verursachen. Bei der Schwellwertvariation ist der Grauwert von 166 als Optimum festgestellt worden. Diese Filterantwort ist in Bild 7 aufgezeigt. Das Bild zeigt, dass die Halbzeuge bezüglich ihrer inneren und äußeren Konturen vollständig sind.

Neben den Halbzeugen sind partielle Flächen des Schneidkopfs, der Arbeitsraumbegrenzungen und des Anschlags zu erkennen. Als weitere Störgrößen sind die Reflexionen der Lamellen und die Spiegelungen auf der Wasseroberfläche zu nennen. Die aufgezählten Gebiete, ausgeschlossen der Halbzeuge, lassen sich in zwei Gruppen einteilen. Die erste Gruppe sind die statischen und die zweite Gruppe die dynamischen Elemente. Zu den statischen Elementen gehören der Schneidkopf, die Arbeitsraumbegrenzungen und der Anschlag und zu den dynamischen die Reflexionen der Lamellen und der Wasseroberfläche.

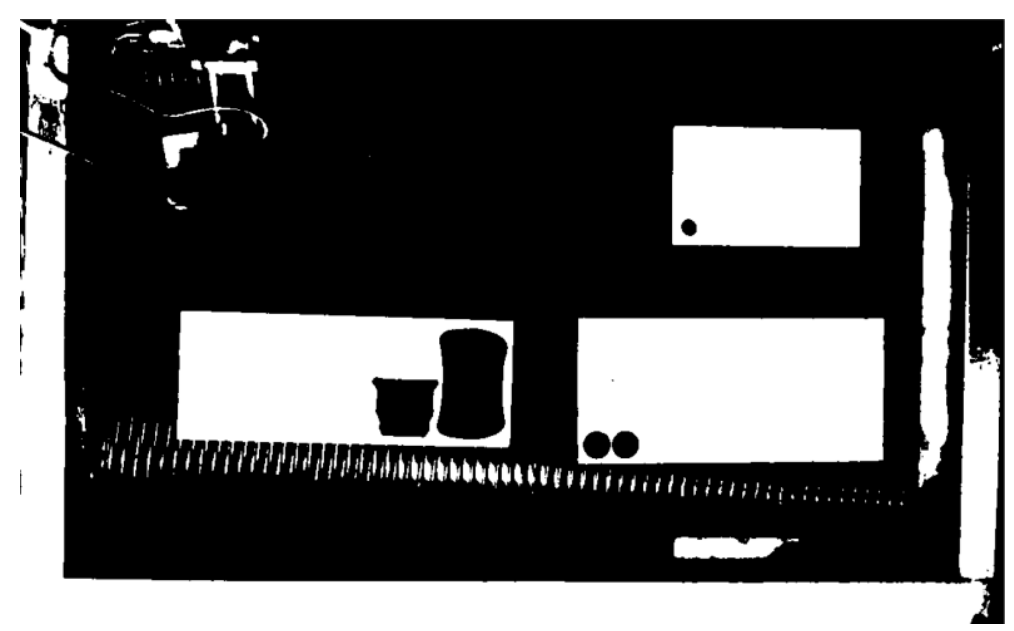

Bild 7: Grauwertmanipulation mit einem Schwellwert von 166

Quelle: eigene Darstellung erstellt mittels Matlab

\subsection{Eliminierung der statischen Elemente}

Die Eigenschaft der Statik ist in diesem Kontext rein räumlich zu verstehen. Die Arbeitsraumbegrenzung inklusive dem Anschlag verschieben sich nicht. Dem Schneidkopf wird per Zwangsbedingung eine feste Position zugewiesen. Die Zwangsbindung lautet, für die nachfolgende Vorgehensweise, dass der Schneidkopf bei der Bildaufnahme in Parkposition zu stehen hat. Durch die feste Zuweisung von Positionen für die jeweiligen Elemente ist es möglich durch einfache Subtraktion diese aus der Filterantwort zu löschen. Dabei tritt die Filterantwort aus Bild 7 als Minuend auf und eine manipulierte Bildaufnahme bestehend aus allen statischen Elementen als Subtrahend. Die gewonnene Differenz besteht folglich nur noch aus den Halbzeugen und den dynamischen Elementen, siehe Bild 8.

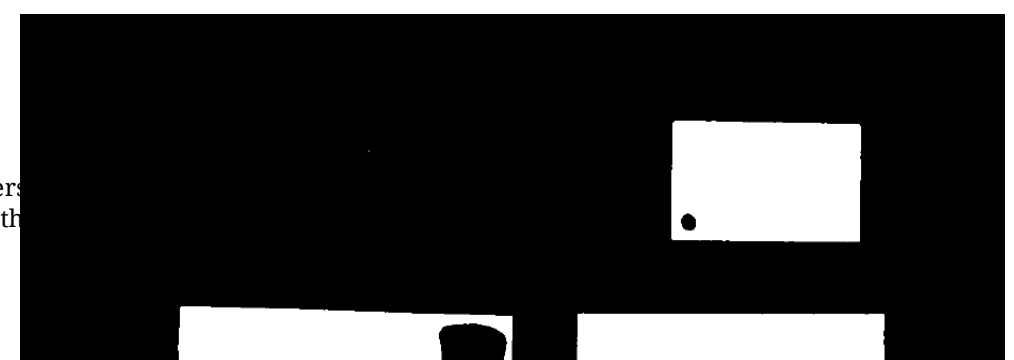




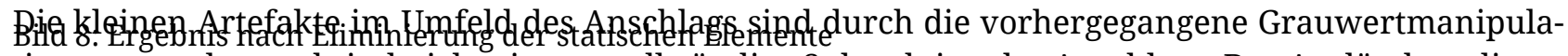

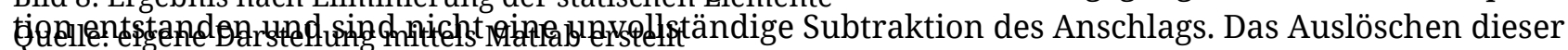
Artefakte und der dynamischen Gebiete ist der nächste Arbeitsschritt.

\subsection{Eliminierung der dynamischen Elemente}

Die Herausforderung der dynamischen Objekte besteht darin, dass sie nicht wie der Schneidkopf, per Zwangsbedingung statisch gestaltbar sind. Sie sind abhängig von verschiedenen Störgrößen, wie Beleuchtung oder Wasserstand, im Arbeitsraum. Dadurch ist eine allgemeine Subtraktion ausgeschlossen. Ebenso ist durch das Schwellwertverfahren aus Unterkapitel 2.2, eine weitere Manipulation der Grauwerte nicht mehr möglich, da die Bildmatrix nur noch aus Einträgen mit 255 oder 0 besteht.

Wird das Bild 8 näher betrachtet fällt auf, dass die Flächen der dynamischen Elemente gegenüber der Halbzeugflächen kleiner sind. Die dynamischen Artefakte bestehen folglich aus weniger Pixeln, was ein Unterscheidungsmerkmal darstellt. Die erstellte Eliminierung baut auf diesen Unterschied auf und filtert Flächen bestehend aus einer bestimmten Pixelanzahl heraus. Bei der Suche nach der richtigen Grenze für die Pixelanzahl wurden verschiedene Größenordnungen getestet. Das Ergebnis der Suche ergab einen Grenzwert von 201 Pixeln. Alle Flächen die kleiner gleich 201 Pixeln sind werden herausgefiltert. Das Ergebnis ist in Bild 9 abgebildet.

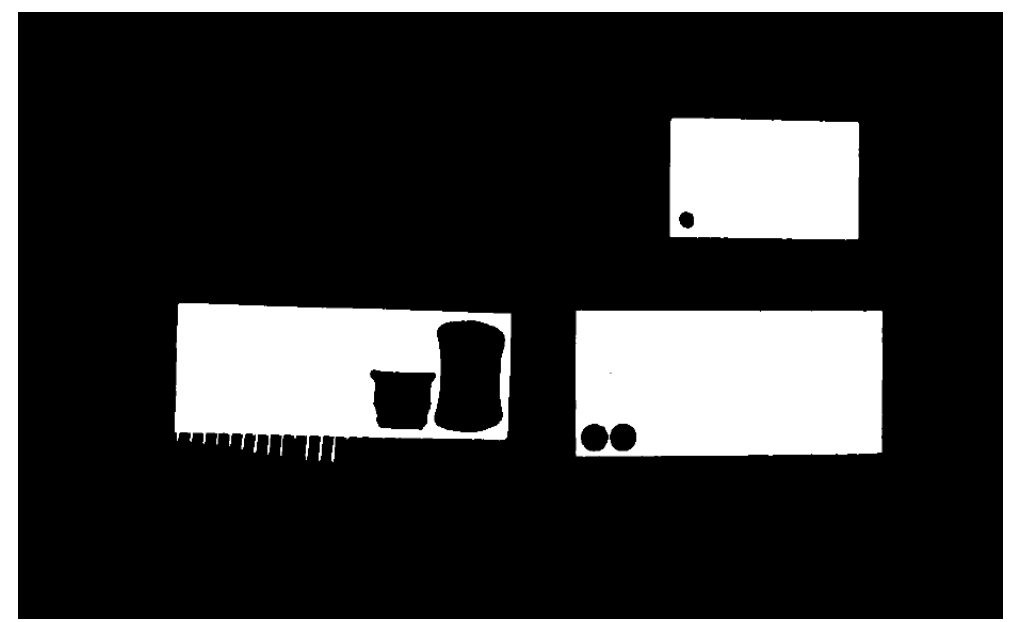

Bild 9: Eliminierung aller dynamischen Elemente mit einer Größe $\leq 201$ Pixeln

Quelle: eigene Darstellung erstellt mittels Matlab

Durch diese Unterdrückung entsteht eine mögliche Fehlerquelle, da auch Halbzeuge in derselben Größenordnung unterdrückt werden würden. Wird die Struktur der Reflexionsartefakte betrachtet, fällt auf, dass es sich dabei um den Bereich zwischen zwei Lamellen oder um Reflexionen entlang der Lamellen handelt. Daraus folgt, dass die Artefakte in ihrer Fläche durch die Lamellen begrenzt sind. Da die Lamellen die Ablagefläche für das Halbzeug darstellen, ist ein Halbzeug, welches kleiner ist als der 
Abstand zweier Lamellen, auf dieser Anlage ohnehin ohne Vorkehrungen nicht bearbeitbar. Die Pixelgrenze von 201 ist somit praktikabel und ein tolerierbarer Fehler. Für den Sonderfall kleinerer Halbzeuge steht weiterhin die manuelle Einrichtmethode zur Verfügung. Nach der aufgezeigten Filtermethodik bleibt mit den Artefakten, die mit dem Halbzeug verschmelzen, ein Problem bestehen. Durch die vorangegangenen Arbeitsschritte liegt ein Schwarzweißbild vor, sodass numerisch keine Separation mehr möglich ist. Die Manipulation entlang des direkten Signalflusspfades ist daher nicht mehr zielführend. Aufgrund dessen wird folgend die Alternative in Form einer Parallelschaltung näher untersucht.

\section{Parallelschaltung}

Die zugrundeliegende Idee ist anstatt einer Bildaufnahme eine Differenzgröße zu betrachten. Im Kunstbereich, wie auch bei Bildbeschreibungen, sind die Wörter Vordergrund und Hintergrund sehr geläufig. In diesem Kapitel werden diese Bezeichnungen ebenfalls eingeführt. Im Duden ist der Hintergrund definiert als „hinterer, abschließender Teil des Blickfeldes bzw. des im Blickfeld liegenden Raums, Bereichs (von dem sich die Gegenstände abheben)“ [9]. Ein Bereich von dem sich Gegenstände abgrenzen, ist die hier genutzte Bedeutung, weshalb der Arbeitsraum fortan als Hintergrund definiert wird. Das bedeutet, wird ein Bild aufgenommen, wo kein Halbzeug im Arbeitsfeld liegt, illustriert dieses Bild den vollständigen Hintergrund. Demzufolge sind alle zu bearbeitenden Materialen, die in den Arbeitsraum gelegt werden, der Vordergrund. Damit liegt das Interesse nur am Vordergrund und Ziel ist die vollständige Eliminierung des Hintergrundes.

\subsection{Eliminierung des Hintergrundes}

Da der Hintergrund durch eine Aufnahme des leeren Arbeitsraumes verkörpert wird, ist der erste Schritt die Differenzbildung des vollen und leeren Arbeitsraumes. Die erhaltene Differenz ist als Vergleichsgröße zu verstehen, da alle übereinstimmenden Charakteristika ausgelöscht werden. Die Aufnahme des leeren Arbeitsraumes ist daher kurz vor dem Ablegen von Halbzeugen zu generieren, sodass die dynamischen Elemente ein Maximum an Übereinstimmung besitzen. Wird eine solche Differenz von Bild 3 erschaffen, entsteht Bild 10.

\section{2 Überführung in Graustufen \& Einsatz von Strukturelementen}

Die erstellte Vergleichsgröße, siehe Bild 10, ist ein Bildtensor, welche durch bereits angewandte Algorithmen aus Kapitel 2 in eine Bildmatrix überführt wird. Ebenso wird das Schwellwertverfahren wieder angewendet. Anzumerken ist, dass der Schwellwert aufgrund der vorherigen Differenzbildung von 166 auf 80 angepasst wurde.

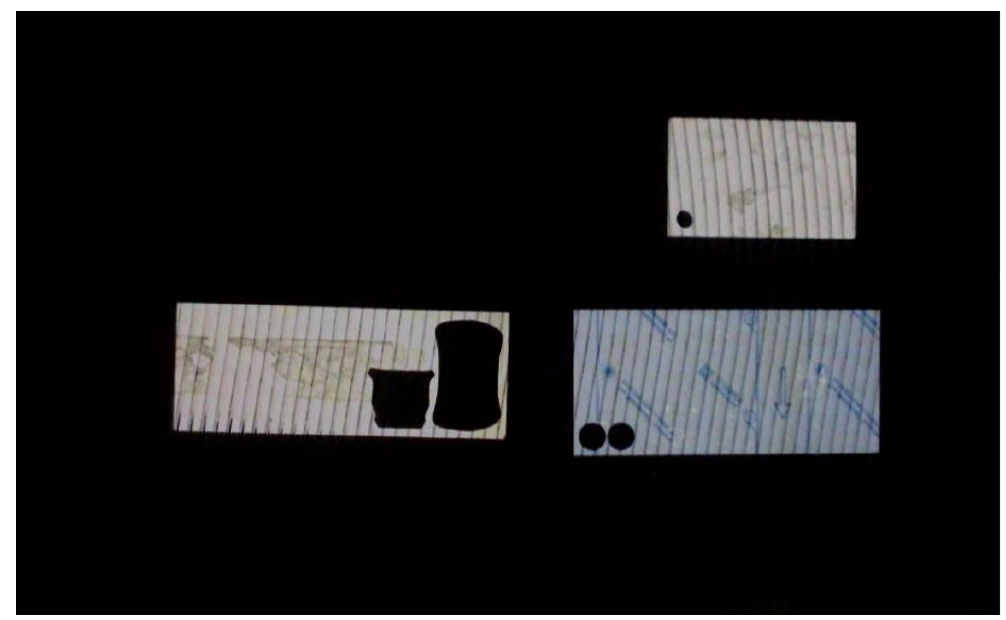


Bild 10: Ergebnis Differenzmethode

Quelle: eigene Darstellung erstellt mittels Matlab

Die größte Herausforderung in diesem Kontext besteht in dem Schließen der lamellenförmigen Defizitstellen. Diese wird durch den Einsatz von Strukturelementen angegangen. Für diesen Anwendungsfall werden zunächst zwei Linienelemente erstellt, da diese die größte Ähnlichkeit zur Lamellenkontur besitzen. Da die Lamellen nicht linear verlaufen, werden die zwei verschieden orientiert, um die Krümmung der Lamellenkontur anzunähern, siehe Bild 11. Lediglich im vorderen rechten Halbzeug sind winzige Defizite zu erkennen, welche zur einfacheren Erkennung rot umkreist sind. Diese sind mit einem weiteren Strukturelement, dem einer Raute, schließbar, siehe Bild 12. Abschließend ist Bild 12 mithilfe des Kirschoperators auf seine Kanten zu reduzieren. Das Filterergebnis ist Bild 13 zu entnehmen.

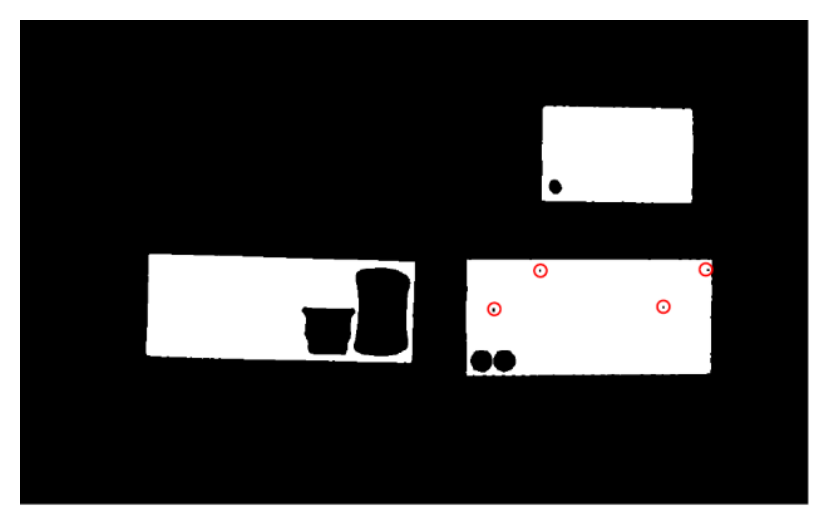

Bild 12: Vergleichsgröße mit Linienelementen Quelle: eigene Darstellung erstellt mittels Matlab

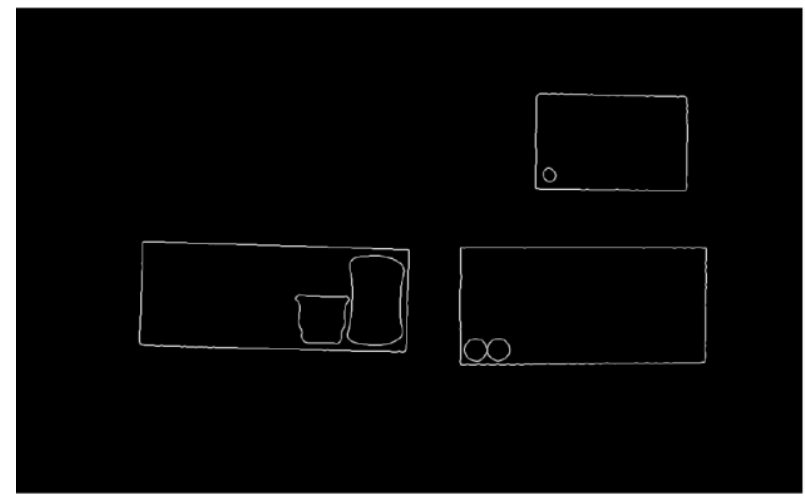

Bild 13: Merkmalsbild generiert durch Kirschfilter Quelle: eigene Darstellung erstellt mittels Matlab

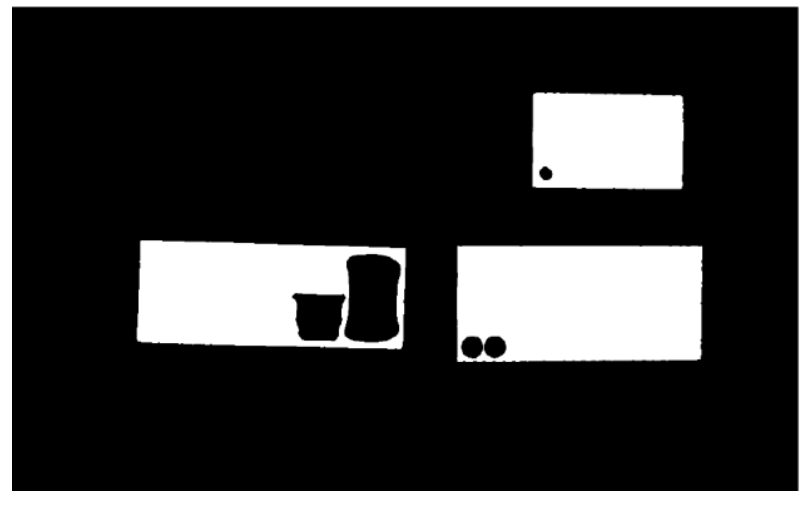

Bild 11: Vergleichsgröße mit zusätzlichen Rautenelementen

Quelle: eigene Darstellung erstellt mittels Matlab

\section{Testen der Parallelschaltungsalgorithmik}

Bild 13 zeigt, dass das gesetzte Etappenziel der Automatisierungsidee erreicht ist. Jedoch ist dies nur erreicht, wenn der gefundene Algorithmus nicht nur für Bild 3, sondern für ein x-beliebiges Bild des Arbeitsraumes funktioniert. Daher ist zu testen, ob die Robustheit des Programmes ausreicht. Dafür wird ein Testbild, Bild 14, herangezogen, welches den Manipulationsprozess aus Kapitel 3 durchläuft. Das Ergebnis ist in Bild 15 dargestellt. Neben dem Testbild, Bild 14, welches exemplarisch aufgezeigt 
ist, wurde das Programm auch mit über 50 weiteren Bildern getestet. Das Ergebnis lautet: Der Algorithmus funktioniert einwandfrei für das Eingangsbild, an welchem er entwickelt wurde. Bei Veränderung des Eingangsbildes ist der Algorithmus jedoch manuell anzupassen.

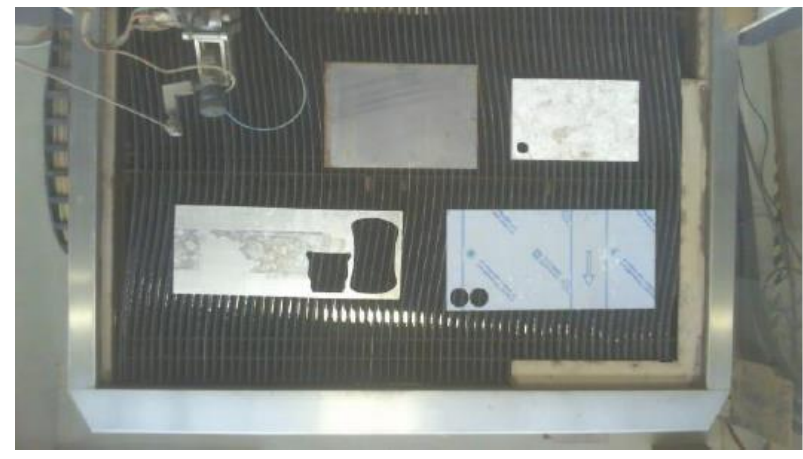

Bild 14: Testbild

Quelle: Umfeldsensorik

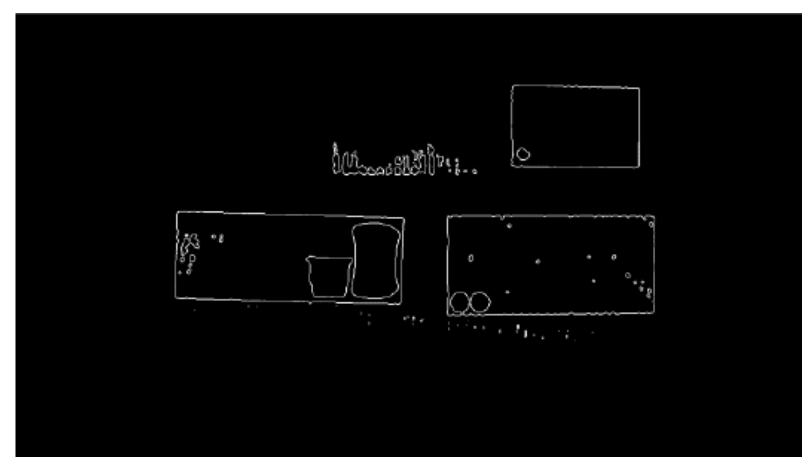

Bild 15: Ergebnisbild

Quelle: eigene Darstellung erstellt mittels Matlab

\section{Schlussfolgerungen \& Diskussion}

Die Komplexität und die Vielzahl an Störgrößen minimieren die Robustheit. Der Algorithmus funktioniert für ein bestimmtes Eingangsbild und ist nur durch Anpassung auf weitere Bilder übertragbar. Auch die Optimierung durch verschiedene Grenzwertverfahren, Schließungen durch Strukturelemente, Unterdrückung kleinerer Artefakte und die Anwendung der Differenzmethode reichen nicht aus, um die gewünschte Prozesssicherheit zu erreichen.

Die Zeitvarianz des Systems erschwert darüber hinaus, die Erstellung eines Lösungskonzeptes. Die benötigten Entscheidungsprozesse, die bei der Separation von Halbzeug und Arbeitsraum nötig sind, sind für den Menschen intuitiv, jedoch für die Maschine teilweise nicht möglich. Eine teils numerische exakte Übereinstimmung der Grauwerte unterbindet die Trennungsmöglichkeit durch die klassischen Kantenoperatoren, weshalb zukünftig das Feld der KI erschlossen wird.

\section{Förderhinweis}

Das Forschungsprojekt wird gefördert durch das Bundesministerium für Wirtschaft und Energie.

| Bundesministeritim
für Wirtshhaft
und Technologie

\section{Literatur}

[1] Muhs S., Meinel G., Burghardt D.; Dezember 2012; Konzept und Ergebnisse einer automatisierten Baublockabgrenzung aus topographischen Kartenwerken; KN - Journal of Cartography and Geographic Information; S. 145-150; URL: https://doi.org/10.1007/BF03544489 (letzter Zugriff am 19.11.2021)

[2] Technische Hochschule Ostwestfalen-Lippe; Lemgo; Labor für Fabrikautomatisierung; URL: https:/www.thowl.de/produktion/fachbereich/labore/fabrikautomatisierung/technologie/ (letzter Zugriff am 08.12.2021)

[3] Fritz A. H. \& Schulze G. (Hrsg.); 2008; Fertigungstechnik; Springer Berlin Heidelberg; URL:https://doi.org/10.1007/978-3-540-76696-4 (letzter Zugriff am 19.11.2021) 
[4] Chatterjee S., Suman A., Gaurav R., Banerjee S., Singh A. K., Ghosh B. K., Mandal R. K., Biswas M. \& Maji D.; Januar 2021; Retinal Blood Vessel Segmentation Using Edge Detection Method; Journal of Physics: Conference Series 1717 012008; URL: https://doi.org/10.1088/1742-6596/1717/1/012008 (letzter Zugriff am 19.11.2021)

[5] Gao P., Sun X. \& Wang W; 2010; Moving object detection based on kirsch operator combined with Optical Flow; 2010 International Conference on Image Analysis and Signal Processing; S. 620-624; URL:https://doi.org/10.1109/IASP.2010.5476045 (letzter Zugriff am 19.11.2021)

[6] Aparna S., Naidu M.E.; Juli 2016; Dynamic Edge Detection in a Digital Video Stream Using Kirsch Filters; Internationale Zeitschrift für Informatik und Informationssicherheit (IJCSIS),vol. 14, Nr. 7; Juli 2016; S. 871878; URL: http://dx.doi.org/10.13140/RG.2.2.25920.87049 (letzter Zugriff am 09.12.2021)

[7] Burger W. \& Burge M. J.; 2015; Digitale Bildverarbeitung; Springer Berlin Heidelberg; URL:https://doi.org/10.1007/978-3-642-04604-9 (letzter Zugriff am 19.11.2021)

[8] Zamperoni P.; 1989; Methoden der digitalen Bildsignalverarbeitung; Vieweg+Teubner Verlag; URL:https://doi.org/10.1007/978-3-322-83935-0 (letzter Zugriff am 21.11.2021)

[9] Nachschlagwerk: Duden | Hintergrund | Rechtschreibung, Bedeutung, Definition, Herkunft; URL:https://www.duden.de/rechtschreibung/Hintergrund (letzter Zugriff am 01.12.2021) 